\title{
Morphology of Mouse Anterior Cruciate Ligament-Complex Changes Following Exercise During Pubertal Growth
}

\author{
Stephen H. Schlecht ID $^{1,2}$ Colin T. Martin, ${ }^{3}$ Danielle N. Ochocki, ${ }^{3}$ Bonnie T. Nolan, ${ }^{1}$ Edward M. Wojtys, ${ }^{1}$ \\ James A. Ashton-Miller ${ }^{2}$ \\ ${ }^{1}$ Department of Orthopaedic Surgery, University of Michigan, Ann Arbor, Michigan, ${ }^{2}$ Department of Mechanical Engineering, University of \\ Michigan, Ann Arbor, Michigan, ${ }^{3}$ Department of Chemistry, University of Michigan, Ann Arbor, Michigan
}

Received 27 August 2018; accepted 8 April 2019

Published online in Wiley Online Library (wileyonlinelibrary.com). DOI 10.1002 /jor.24328

\begin{abstract}
Postnatal development and the physiological loading response of the anterior cruciate ligament (ACL) complex (ACL proper, entheses, and bony morphology) is not well understood. We tested whether the ACL-complex of two inbred mouse strains that collectively encompass the musculoskeletal variation observed in humans would demonstrate significant morphological differences following voluntary cage-wheel running during puberty compared with normal cage activity controls. Female A/J and C57BL/6J (B6) 6-week-old mice were provided unrestricted access to a standard cage-wheel for 4 weeks. A/J-exercise mice showed a 6.3\% narrower ACL $(p=0.64)$, and a $20.1 \%$ more stenotic femoral notch $(p<0.01)$ while B6-exercise mice showed a $12.3 \%$ wider ACL $(p=0.10)$, compared with their respective controls. Additionally, A/J-exercise mice showed a 5.3\% less steep posterior medial tibial slope ( $p=0.07)$ and an $8.8 \%$ less steep posterior lateral tibial slope $(p=0.07)$, while B6-exercise mice showed a 9.8\% more steep posterior medial tibial slope $(p<0.01)$ than their respective controls. A/J-exercise mice also showed more reinforcement of the ACL tibial enthesis with a 20.4\% larger area $(p<0.01)$ of calcified fibrocartilage distributed at a $29.2 \%$ greater depth $(p=0.02)$ within the tibial enthesis, compared with their controls. These outcomes suggest exercise during puberty significantly influences ACL-complex morphology and that inherent morphological differences between these mice, as observed in their less active genetically similar control groups, resulted in a divergent phenotypic outcome between mouse strains. (C) 2019 Orthopaedic Research Society. Published by Wiley Periodicals, Inc. J Orthop Res 37:1910-1919, 2019
\end{abstract}

Keywords: ACL; fibrocartilage; knee; ligament; bone

The impact of physiological loading on the postnatal development of the anterior cruciate ligament (ACL) complex (i.e., the ACL proper, entheses, and bony morphology) is not well understood. An increase in the strength and/or stiffness of the ACL in response to exercise throughout growth has been reported in animal models. ${ }^{1,2}$ Viidik $^{1}$ showed that high-frequency exercise in skeletally immature rabbits increased ACL strength, particularly at the tibial entheses, while Cabaud et al. ${ }^{2}$ demonstrated that endurance exercise increased ACL interstitial strength and stiffness in growing rats. However, neither of these studies reported on the influence exercise during musculoskeletal growth has on the morphological traits encompassing the ACL-complex. This includes ACL size, the calcified fibrocartilaginous matrix within the femoral and tibial entheses, as well as the distal femoral and proximal tibial epiphyseal morphology comprising the knee joint. To date, Grzelak et al. ${ }^{3}$ provide the only known report attributing activity to a 3.5-fold larger ACL crosssectional area among high-performance weightlifters that began training as adolescents compared with agematched controls. However, this magnetic resonance study included only nine athletes and was dependent on self-reporting.

Grant sponsor: NIH National Institute of Arthritis and Musculoskeletal and Skin Diseases; Grant number: SHS: AR070903; Grant sponsor: Michigan Integrative Musculoskeletal Health Core Center; Grant number: NIH/NIAMS: AR065424. Correspondence to: Stephen H. Schlecht (T: 734-647-1528;

F: 734-647-0003; E-mail: sschlech@med.umich.edu)

(c) 2019 Orthopaedic Research Society. Published by Wiley Periodicals, Inc.
Clinically, associations between several morphological traits of the knee and ACL-injury risk have been widely reported in both sexes across various ages and sports activities. These morphological risk factors, which in vitro studies have found to increase the likelihood of ACL impingement, ${ }^{4}$ anterior tibial translation $^{5,6}$ and/or increased ACL peak strains, ${ }^{7,8}$ include ACL cross-sectional area, ${ }^{7,9}$ femoral intercondylar notch width/shape,$^{10,11}$ and posterior tibial plateau slope. ${ }^{12,13}$ How the morphology of these anatomical traits is biomechanically influenced throughout growth via differentially applied physiological loads is of considerable clinical importance yet remains unclear. Interestingly, Tuca et al. ${ }^{14}$ recently reported their findings from a magnetic resonance imaging study conducted on boys and girls ranging from 3 to 14 years old that suggested the significant growth in ACL volume and femoral intercondylar notch volume plateaued by 10 years of age in both sexes. Their finding supports the prevailing dogma of the field that these morphological traits are largely non-modifiable after the pre-pubescence phase. ${ }^{15}$ However, the findings of Tuca et al. ${ }^{14}$ research is inconsistent with other musculoskeletal studies conducted on high activity individuals. Bone ${ }^{16-18}$ muscle,${ }^{19-21}$ and tendinous size $^{22,23}$ are significantly different morphologically in the dominantly loaded musculoskeletal element of individuals compared with their non-dominant contralateral and in comparison with individuals that had a lower activity level throughout growth (both pre- and post-puberty). ${ }^{3,16,24-28}$

In light of the uncertainty surrounding how the knee joint and associated ACL traits are affected following 
increased mechanical loading during postnatal growth, we tested two hypotheses using a mouse model $(\mathrm{A} / \mathrm{J}$ and C57BL/6J) with a phenotypically similar knee structure as humans. ${ }^{29}$ Our previous pilot work found that A/J mice exhibit a slender knee joint morphology with narrow femoral and tibial condyles, a stenotic intercondylar femoral notch, a narrow ACL, and a narrow tibial plateau. They also have moderately sloped medial and lateral posterior tibial plateaus and broad yet shallow ACL entheses (i.e., uncalcified and calcified fibrocartilaginous zones demarcated by a tidemark that functionally anchor the ligament into bone). On the other hand, C57BL/6J (B6) mice have a robust knee joint morphology with wide femoral and tibial condyles, a parabolic intercondylar femoral notch, a wide ACL, and a broad tibial plateau. Additionally, they have severely sloped medial and lateral posterior tibial plateaus and narrow yet deep ACL entheses. Moreover, $\mathrm{A} / \mathrm{J}$ and $\mathrm{C} 57 \mathrm{BL} / 6 \mathrm{~J}$ (B6) mice have differing knee joint mechanics with $\mathrm{A} / \mathrm{J}$ mice previously shown having a $68 \%(p<0.001)$ greater joint laxity and a lower $(p=0.003)$ toe region stiffness compared with B6 mice at musculoskeletal maturity. ${ }^{30}$ Using this model, which collectively encompasses the variation in knee joint morphology observed across male and female humans, ${ }^{7,13,31,32}$ we first tested the hypothesis that pubescent (6-10 weeks of age) exercised mice of both inbred strains would show significant phenotypic differences in their ACL-complex morphology, particularly in regards to ACL cross-sectional area and intercondylar notch width, compared with their normal cage activity controls. Additionally, since the knee joint phenotype naturally differs between $\mathrm{A} / \mathrm{J}$ and $\mathrm{B} 6$ mice we also tested the hypothesis that the resulting outcome 4 weeks of increased physiological loading would have on traits comprising the ACL-complex would be different between these two mouse strains. This was tested by defining the post-exercise differences in ACL size, calcified fibrocartilage (CF) distribution (reflective of ACL bone anchorage footprint), and bone morphology between mouse strains.

Previously, we reported a significantly larger bone mass in the femoral $(p=0.01)$ and humeral $(p=0.02)$ diaphyses and distal femora $(p=0.02)$ following voluntary exercise between 8 -week-old $\mathrm{A} / \mathrm{J}$ females compared with their controls. This morphological change was not observed in their exercised B6 counterparts. ${ }^{33}$ Based on these outcomes, we also hypothesized that the gracile knee of A/J mice would show a greater expansion of the intercondylar notch, ACL, and CF within the femoral and tibial ACL entheses at 10 weeks of age following exercise when compared with exercised B6 mice.

Characterizing the morphology of ACL-complex traits following an increase in pubescent activity will shed light on how an increase in physiological loading during a critical musculoskeletal growth phase may or may not influence the resulting adult knee morphology. Moreover, it will help us to better understand indirectly the influence of physiological loading upon the
ACL-complex may have on the inherent baseline metabolic $^{34,35}$ and musculoskeletal ${ }^{36,37}$ differences that exist between $\mathrm{A} / \mathrm{J}$ and $\mathrm{B} 6$ mice. The overarching clinical importance of this study is to determine whether it is feasible for ACL-complex morphological traits commonly associated with ACL-injury risk to be biomechanically susceptible to an increase in physical activity during the pubescent growth phase.

\section{METHODS}

Choice of age at entry and duration of the study were chosen to coincide with the hormonal fluctuations and rapid skeletal growth occurring during murine puberty. Six weeks of age in mice is the average at which puberty begins, ${ }^{38,39}$ and 10 weeks of age is the average at which mice reach sexual maturity-a biological indicator of adulthood. ${ }^{40}$ Twenty-four female A/J and 24 female B6 inbred mice were purchased from the Jackson Laboratory (Bar Harbor, ME) at 5 weeks of age and allowed 1 week to acclimate before the start of the study. For each strain, mice were block randomized into a control $(n=12)$ or exercise group $(n=12)$. Mice were distributed into each group using body weight (BW) to ensure that all groups were similar in size at the start of the study $(p>0.50)$. Mice in both the control and exercise groups were individually housed for the duration of the study. All mice were provided water and fed a standard rodent diet (D12450B; Research Diets, New Brunswick, NJ) ad libitum. Mice were kept on a 12-h light/dark cycle and provided a nestlet for cage enrichment. A/ $\mathrm{J}$ and B6 mice assigned to the exercise group ( $n=12 /$ strain) had free access $(24 \mathrm{~h} /$ day $)$ to a stainless-steel cage-wheel (115 mm outer diameter; Mini-Mitter Co., Inc., Murrysville, PA) for 4 weeks. Wheel revolutions were monitored daily, and the distance run $(\mathrm{km})$ was calculated as the number of revolutions $\times$ the outer diameter of the wheel $\times \pi$. Control mice were allowed normal cage activity during the study. BW and food weight $(\mathrm{FW})$ were recorded one time per week throughout the course of the experiment. Mice were euthanized at 10 weeks of age and the left and right legs ( $n=12 /$ group/strain) were harvested, cleaned of all soft tissue leaving only the knee joint intact, and stored in $1 \times$ phosphate-buffered saline (PBS) solution at $-40^{\circ} \mathrm{C}$. The Institutional Animal Care and Use Committee (IACUC) at the University of Michigan approved all handling and treatment of mice for this study. Table 1 defines trait abbreviations that are frequently used throughout the article.

\section{ACL Morphology}

The knee joint of the left and right legs from both control and exercise mice were dissected using a Leica S6E stereo microscope equipped with a Leica EC3 digital color camera (Leica Microsystems, Inc., Buffalo Grove, IL). The infrapatellar fat pad, collateral ligaments, menisci, and posterior cruciate ligament were carefully removed, leaving only the ACL intact. Due to the small and relatively fragile nature of the specimens, the posterolateral and anteromedial bundles of the ACL could not be separated. Following dissection, the knee was imaged in the coronal and sagittal planes using the stereo microscope for quantification of the maximum posterior length (ACL.Post.Le) and posterior and medial widths (ACL.Post.Wi and ACL.Med.Wi) of the ACL. Measurements were taken using ImageJ (NIH) to calculate ellipsoidal crosssectional area (ACL Ell.CSA). 
Table 1. Frequently Used Abbreviations

\section{Femoral measures}

ACL Ell.CSA: ACL elliptical cross-sectional area Fem.Le: Maximum femur length

ANWI: Anterior femoral notch width index CNWI: Central femoral notch width index PNWI: Posterior femoral notch width index NSI: Femoral notch shape index

Fem.Bicon.Wi: Femoral bicondylar width

Fem.TM.Le: Femoral enthesis tidemark length

Fem.CF.De: Femoral calcified fibrocartilage depth

Fem.CF.Ar: Femoral calcified fibrocartilage area

Tibial measures

Tib.Le: Maximum tibial length

Tib.Bicon.Wi: Tibial bicondylar width

PMTS: Posterior medial tibial plateau slope

PLTS: Posterior lateral tibial plateau slope

Tib.TM.Le: Tibial enthesis tidemark length

Tib.CF.Ar: Tibial calcified fibrocartilage area

Tib.CF.De: Tibial calcified fibrocartilage depth

\section{Knee Joint Morphology}

After imaging the ACL for areal quantification, the legs were fixed for $72 \mathrm{~h}$ in $10 \%$ neutral buffered formalin. Upon fixation, each ACL was transected midsubstance for three-dimensional imaging of the femora and tibiae of each mouse. All bones were imaged while submerged in $1 \times$ PBS at an $8-\mu \mathrm{m}$ voxel size using nano-computed tomography (nanotom-s; GE Sensing and Inspection Technologies, GmbH, Wunstorf, Germany). Imaging parameters were set to $90 \mathrm{kV}, 375 \mu \mathrm{A}, 1,000 \mathrm{~ms}$, three averages and one skip, with a $0.3-\mathrm{mm}$ aluminum filter. Gray values were converted to Hounsfield units using a calibration phantom containing air, water, and a hydroxyapatite mimicker (1.69 mg/cc; Gammex, Middleton, WI) as described previously. ${ }^{37}$
Image analysis was conducted using Microview Advanced Bone Analysis (v. 2.2; GE Healthcare) and VGStudio (Volume Graphics, Inc., Charlotte, NC). Maximum femoral (Fem.Le) and tibial (Tib.Le) lengths were measured from the image stacks. For femoral analysis, bones were anatomically reoriented, and the slices used for analysis were standardized based on morphological landmarks. Several measurements of the intercondylar femoral notch were made in accordance with Fitch et al. ${ }^{41}$ and Comerford et al. ${ }^{42}$ These measures included the anterior (Ant.Not.Wi), central (Cen.Not.Wi), and posterior (Post.Not.Wi) notch widths, intercondylar notch height (Not.Ht), and bicondylar width (Fem.Bicon.Wi) (Fig. 1A). Variables derived from these measures included notch width indices (i.e., ANWI: Ant.Not.Wi/Fem.Bicon.Wi; CNWI: Cen.Not.Wi/Fem.Bicon.Wi; and PNWI: Post.Not.Wi/Fem.Bicon.Wi) and an estimate of the femoral notch shape (NSI: Cen.Not.Wi/Not.Ht).

Similar to the femora, tibiae were reoriented, and slices used for analysis were defined based on morphological landmarks. Methods used for obtaining measurements from the tibial plateau were in accordance with Reif and Probst. ${ }^{43}$ Briefly, the longitudinal axis of the tibia was defined as the middle of the tibial intercondylar eminences through the center of the talar articular surface. A line perpendicular to this axis was then defined along the medial and lateral plateau surfaces. The angle between the posterior medial and lateral plateau surfaces and the perpendicular line was quantified, providing the degree of the posterior medial (PMTS) and posterior lateral (PLTS) tibial slope for each tibia (Fig. 1B). The bicondylar width of the tibial plateau (Tib.Bicon.Wi) was also measured.

\section{Entheseal Histomorphometry}

Following three-dimensional imaging of the femora and tibiae, all bones were decalcified in $10 \%$ ethylenediaminetertraacetic acid for 10 days, dehydrated through graded alcohols, and embedded in paraffin. For the femoral and tibial blocks, sagittal $7-\mu \mathrm{m}$ serial sections were taken across the entire ACL

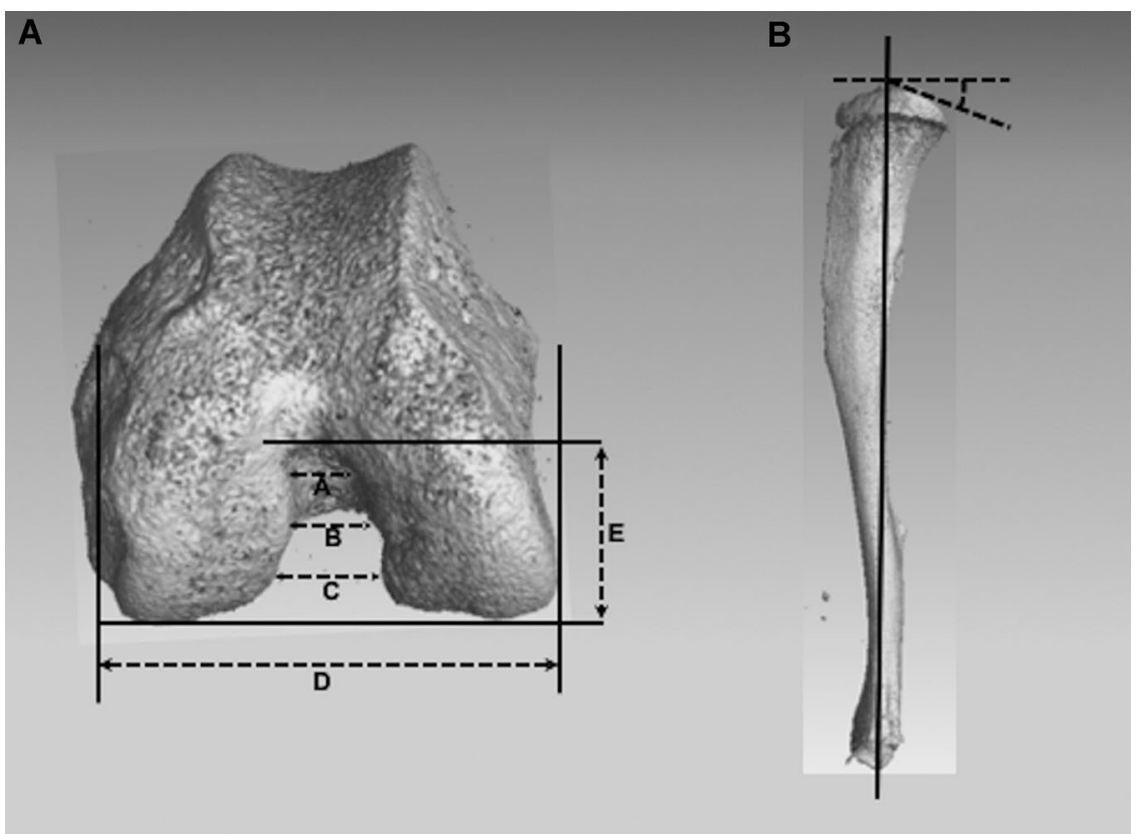

Figure 1. Bone morphology measurements. (A) Schematic of distal femoral epiphyseal anthropometric measurements. A, Ant.Not. Wi; B, Cen.Not.Wi; C, Post.Not.Wi; D, Tt. Con.Wi; and E, Not.Ht. (B) Schematic of proximal tibial epiphyseal anthropometric measurements. Solid line represents the longitudinal axis of the tibia with the dotted lines representing the perpendicular and posterior tibial slope. NanoCT images at an $8 \mu \mathrm{m}$ voxel size. [Color figure can be viewed at wileyonlinelibrary.com] 
enthesis. Paraffin sections were then stained with $1 \%$ toluidine blue (Sigma-Aldrich, St. Louis, MO), a monochromatic stain that highlights the four matrix zones of the ACL enthesis (i.e., ligament proper, uncalcified fibrocartilage [UF], $\mathrm{CF}$, and bone). Stained histologic sections were imaged at $\times 10$ magnification under polarized light using a Nikon Eclipse $(\mathrm{Ni}$; Nikon, Melville, NY) microscope affixed with a digital color camera (DS-Ri2; Nikon). Using NIS Elements software (Nikon), the length of the tidemark (TM.Le), calcified fibrocartilage area (CF.Ar), and the depth of the calcified fibrocartilage (CF.De) were measured as they are reliably quantifiable and directly associated with the anchorage of the ACL into the bone. TM.Le was defined as the total expanse of the basophilic line running from the anterior to the posterior margins of the ACL insertion ${ }^{32}$ which provides a measure of ACL insertion width. CF.Ar was defined as the differentially stained region between the TM and cortical bone. CF.De was defined as the perpendicular distance from the TM to the edge where the CF meets the cortical bone. To account for the heterogeneity in CF.De across the enthesis, measurements were taken at $30 \mu \mathrm{m}$ intervals across the enthesis resulting in $\sim 5-8$ zones depending on the expanse of the ACL insertion ${ }^{44}$ (Fig. 2A and B).

\section{Data Analyses}

All data were analyzed using Minitab v.18 (State College, PA) and Prism v.7 (GraphPad Software, La Jolla, CA). A Shapiro-Wilk test was conducted to determine if the data were normally distributed. All data were normalized via linear regression to the respective BW at the sacrifice of each mouse. BW was chosen to adjust the data since a percentage of the total body mass provides an external force during axial loading of the knee joint, ${ }^{45,46}$ and influences ground-reaction force. ${ }^{4,48}$ Adjusted trait values were entered into a two-way analysis of variance to test for strain and treatment (exercise, control) main effects, and strain by treatment interaction with a significance of $p<0.10$. Tukey's multiple comparisons test between exercised and control mice within and between strains were assessed to determine the significance of least square mean differences in the presence of important interactions. Following this, linear regression analysis was used to identify significant associations between BW adjusted phenotypic traits and distance run.

To test for intraobserver reliability when selecting landmarks for measuring ACL.Le, ACL.Wi, PMTS, and PLTS, each of these variables were measured three separate times from all mice included in the study, one month apart. Intraclass coefficient values obtained from this test demonstrated good to excellent reliability for ACL.Le (0.885), ACL.Post.Wi (0.936), ACL.Med.Wi (0.921), and PLTS (0.871). PMTS demonstrated moderate reliability with an ICC value of 0.631 .

\section{RESULTS}

Table 2 is comprised of mean and standard deviation data for each key trait quantified, after adjusting for BW. Table 3 includes linear regression data showing how each trait is associated with the total distance run of each mouse in the study, after adjusting for BW.

\section{Effect of Distance Run on BW}

At the onset of the running study, mice comprising the control and exercise groups for both strains were sorted so there was no significant difference in mean BW both within strain groups (A/J: $p=0.82 ; \mathrm{B} 6: p=0.66)$ and across strain groups (Controls: $p=0.57$; Exercise: $p=0.51$ ). After 4 weeks of running, A/J-exercise mice showed a non-significant $(p=0.19) 3.49 \%$ lower BW compared with controls, while B6-exercise mice significantly gained $13.2 \%$ more weight $(20.9 \pm 1.0 \mathrm{~g}$; $p<0.01)$ than their controls $(18.4 \pm 1.2 \mathrm{~g})$. Despite $\mathrm{A} / \mathrm{J}$-exercise mice ending with less weight and B6exercise mice ending with more weight than their respective controls, both exercise groups consumed significantly more food throughout the study (A/Jexercise: $23 \%, p<0.01$; B6-exercise: $20 \%, p<0.01$ ) compared with their controls.

Over the course of 4 weeks, A/J-exercise mice had a daily average of distance run that was $10 \%$ more $(9.28 \pm 2.1 \mathrm{~km})$ than B6-exercise mice $(8.42 \pm 1.7 \mathrm{~km})$, however this was not a significant difference $(p=0.27)$ (Fig. 3) since at the onset of the study A/J was slower to adjust to the wheel. Both $\mathrm{A} / \mathrm{J}$-exercise and B6-exercise mice ran significantly more in the 4 th week of the study than they did during the 1 st week of the study $(p<0.01$ and $p=0.03$, respectively).

\section{Exercise Effect on Differences in ACL Size}

Without exercise, A/J-control mice at 10 weeks of age had an ACL that was $4.0 \%$ smaller than that of their B6 counterparts $(p=0.87)$. This non-significant difference was primarily driven by B6-control mice having a greater ACL.Le $(p<0.01)$ and ACL.Post.Wi $(p=0.01)$, compared with A/J-control mice. The difference in ACL Ell.CSA between strains was greater in the mice that ran with A/J-exercise mice having an ACL that was $26.7 \%$ smaller $(p<0.01)$ than that of B6-exercise mice. This differential effect between strains is illustrated by the significant strain by treatment interaction $(F=7.61, p=0.01)$. The dichotomy in ACL size is attributed to B6-exercise mice having a $13.5 \%$ significantly larger ACL Ell.CSA than B6-control mice $(p=0.05)$, and $\mathrm{A} / \mathrm{J}$-exercise mice showing a non-significant $(p=0.58) 6.8 \%$ smaller ACL Ell.CSA compared with controls. Interestingly, the more A/J mice ran the

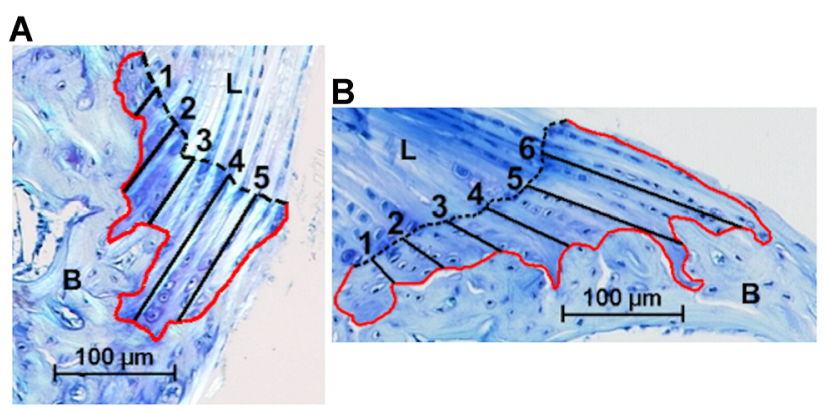

Figure 2. Calcified fibrocartilage measurements for (A) femoral ACL enthesis and (B) tibial ACL enthesis. Dotted black line indicates the tidemark. The red line indicates the boundary between the calcified fibrocartilage and bone. The solid black lines indicate the measurement of calcified fibrocartilage depth at 30 $\mu \mathrm{m}$ intervals along the tidemark. $\mathrm{B}$, bone; $\mathrm{L}$, ligament. Toluidine blue stain at $\times 10$ magnification. [Color figure can be viewed at wileyonlinelibrary.com] 
Table 2. Mean and Standard Deviations of All Key Morphological Traits Quantified After Adjusting for Body Weight*

\begin{tabular}{|c|c|c|c|c|c|c|}
\hline & \multicolumn{3}{|c|}{$\mathrm{A} / \mathrm{J}$} & \multicolumn{3}{|c|}{ B6 } \\
\hline & Control & Exercise & Diff. $(\%)$ & Control & Exercise & Diff. (\%) \\
\hline \multicolumn{7}{|l|}{ Fem. traits } \\
\hline Fem.Le (mm) & $14.40 \pm 0.32$ & $14.30 \pm 0.11$ & -0.69 & $14.87 \pm 0.16$ & $14.60 \pm 0.16$ & -1.85 \\
\hline Fem.Bicon.Wi (mm) & $2.097 \pm 0.037$ & $2.022 \pm 0.089$ & $-\mathbf{3 . 5 7}$ & $2.148 \pm 0.054$ & $2.166 \pm 0.069$ & -0.82 \\
\hline ANWI (mm/mm) & $0.154 \pm 0.023$ & $0.139 \pm 0.018$ & -9.37 & $0.200 \pm 0.020$ & $0.188 \pm 0.031$ & -5.91 \\
\hline CNWI $(\mathrm{mm} / \mathrm{mm})$ & $0.241 \pm 0.025$ & $0.218 \pm 0.018$ & -9.49 & $0.293 \pm 0.0144$ & $0.290 \pm 0.021$ & -0.88 \\
\hline PNWI (mm/mm) & $0.291 \pm 0.013$ & $0.272 \pm 0.023$ & -6.45 & $0.337 \pm 0.015$ & $0.343 \pm 0.015$ & 1.92 \\
\hline NSI $(\mathrm{mm} / \mathrm{mm})$ & $0.665 \pm 0.069$ & $0.530 \pm 0.068$ & -19.06 & $0.696 \pm 0.042$ & $0.665 \pm 0.041$ & -5.89 \\
\hline \multicolumn{7}{|l|}{ Tib. traits } \\
\hline Tib.Le (mm) & $16.53 \pm 0.68$ & $16.59 \pm 0.40$ & -0.35 & $17.20 \pm 0.54$ & $16.64 \pm 0.42$ & -3.27 \\
\hline Tib.Bicon.Wi (mm) & $2.69 \pm 0.028$ & $2.686 \pm 0.025$ & -0.27 & $2.828 \pm 0.031$ & $2.717 \pm 0.054$ & -3.93 \\
\hline $\operatorname{PMTS}\left({ }^{\circ}\right)$ & $25.23 \pm 1.44$ & $24.08 \pm 1.00$ & -4.57 & $26.05 \pm 1.32$ & $28.70 \pm 1.34$ & 10.19 \\
\hline $\operatorname{PLTS}\left({ }^{\circ}\right)$ & $24.32 \pm 1.50$ & $22.34 \pm 2.35$ & -8.15 & $32.46 \pm 2.64$ & $31.33 \pm 1.50$ & -3.47 \\
\hline \multicolumn{7}{|l|}{ ACL traits } \\
\hline ACL Ell.CSA $\left(\mathrm{mm}^{2}\right)$ & $0.060 \pm 0.008$ & $0.056 \pm 0.008$ & -6.98 & $0.062 \pm 0.007$ & $0.071 \pm 0.007$ & 13.55 \\
\hline Fem.TM.Le $(\mu \mathrm{m})$ & $174.2 \pm 13.7$ & $184.0 \pm 28.6$ & 5.59 & $159.1 \pm 15.6$ & $148.6 \pm 9.5$ & -6.61 \\
\hline Fem.CF.Ar $\left(\mu \mathrm{m}^{2}\right)$ & $15,147 \pm 1487$ & $16,387 \pm 1632$ & 8.19 & $15,934 \pm 2390$ & $14,232 \pm 1032$ & -10.68 \\
\hline Fem.CF.De $(\mu \mathrm{m})$ & $84.82 \pm 3.80$ & $83.00 \pm 12.47$ & -2.15 & $103.71 \pm 13.41$ & $99.08 \pm 9.28$ & -4.47 \\
\hline Tib.TM.Le $(\mu \mathrm{m})$ & $347.8 \pm 47.6$ & $284.4 \pm 17.5$ & -18.22 & $219.5 \pm 16.4$ & $222.0 \pm 18.0$ & 1.15 \\
\hline Tib.CF.Ar $\left(\mu \mathrm{m}^{2}\right)$ & $28,863 \pm 5746$ & $22,212 \pm 1217$ & -23.04 & $19,305 \pm 2356$ & $17,093 \pm 1363$ & -11.46 \\
\hline Tib.CF.De $(\mu \mathrm{m})$ & $60.73 \pm 5.45$ & $75.55 \pm 8.87$ & 24.39 & $74.58 \pm 9.77$ & $85.70 \pm 15.85$ & 14.90 \\
\hline
\end{tabular}

"Percent differences are given for the mean difference between the control and exercise group of each strain. Bold values denote significance at $p<0.10$.

Table 3. $R^{2}$ and $p$-Values for Each Trait and Their Association With the Daily Distance Run By Exercise Mice of Each Strain*

\begin{tabular}{lccccc}
\hline & \multicolumn{2}{c}{$\mathrm{A} / \mathrm{J}$} & & \multicolumn{2}{c}{$\mathrm{B} 6$} \\
\cline { 2 - 3 } \cline { 5 - 6 } Trait & $R^{2}$ & $p$ & & $R^{2}$ & $p$ \\
\hline ACL Ell.CSA $\left(\mu \mathrm{m}^{2}\right)$ & $\mathbf{0 . 4 6}$ & $\mathbf{0 . 0 3}$ & & $\mathbf{0 . 4 1}$ & $\mathbf{0 . 0 2}$ \\
Fem.Bicon.Wi $(\mathrm{mm})$ & 0.04 & 0.52 & & 0.02 & 0.88 \\
ANWI $(\mathrm{mm} / \mathrm{mm})$ & 0.16 & 0.22 & & $<0.01$ & 0.82 \\
CNWI $(\mathrm{mm} / \mathrm{mm})$ & $<0.01$ & 0.82 & & $<0.01$ & 0.96 \\
PNWI $(\mathrm{mm} / \mathrm{mm})$ & 0.19 & 0.17 & & 0.02 & 0.65 \\
NSI $(\mathrm{mm} / \mathrm{mm})$ & 0.15 & 0.23 & & 0.13 & 0.27 \\
Fem.TM.Le $(\mu \mathrm{m})$ & 0.26 & 0.20 & $\mathbf{0 . 5 9}$ & $\mathbf{0 . 0 2}$ \\
Fem.CF.Ar $\left(\mu \mathrm{m}{ }^{2}\right)$ & $<0.01$ & 0.93 & & 0.12 & 0.81 \\
Fem.CF.De $(\mu \mathrm{m})$ & $<0.01$ & 0.88 & & 0.08 & 0.53 \\
Tib.Bicon.Wi $(\mathrm{mm})$ & 0.16 & 0.18 & & 0.08 & 0.66 \\
Tib.TM.Le $(\mu \mathrm{m})$ & 0.04 & 0.65 & & 0.01 & 0.71 \\
Tib.CF.Ar $\left(\mu \mathrm{m}^{2}\right)$ & 0.11 & 0.43 & & 0.13 & 0.25 \\
Tib.CF.De $(\mu \mathrm{m})$ & $<0.01$ & 0.97 & & 0.01 & 0.69 \\
PMTS $\left({ }^{\circ}\right)$ & 0.05 & 0.49 & & 0.05 & 0.49 \\
PLTS $\left({ }^{\circ}\right)$ & 0.08 & 0.37 & & 0.13 & 0.27 \\
\hline
\end{tabular}

*Bold values denote significance at $p<0.05$.

smaller ACL Ell.CSA was at 10 weeks of age $\left(R^{2}=0.46\right.$, $p=0.03$ ), whereas the more $\mathrm{B} 6$ mice ran the larger their ACL was at 10 weeks of age $\left(R^{2}=0.41, p=0.02\right)$ (Fig. 4).

Exercise Effect on Knee Joint Morphology

At 10 weeks of age A/J-control mice had a nonsignificant $2.4 \%$ narrower Fem.Bicon.Wi $(p=0.25)$ yet

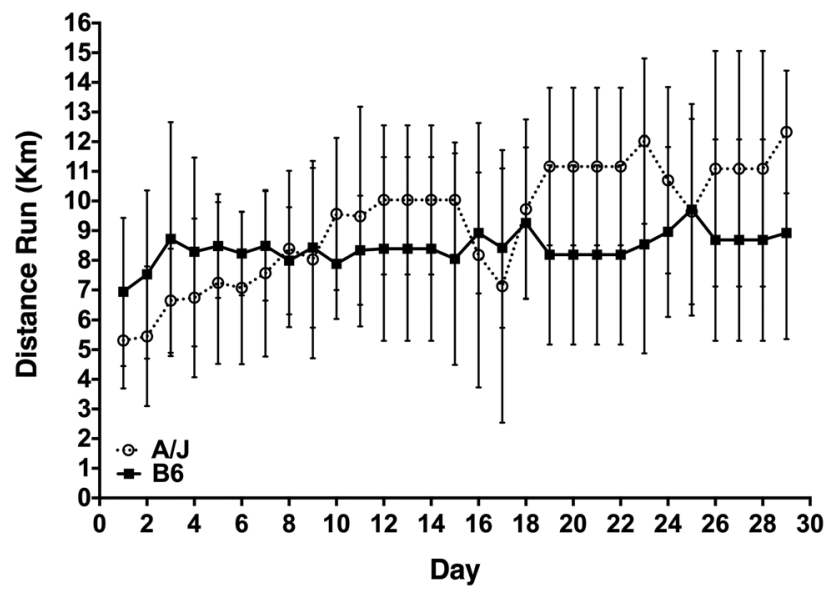

Figure 3. Average and standard deviations of daily distance run by voluntary cage-wheel running mice of both inbred strains throughout the 4-week study. [Color figure can be viewed at wileyonlinelibrary.com]

a significantly narrower femoral notch (ANWI: $-29.8 \%$, $p<0.01$; CNWI: $-21.5 \%, p<0.01$; PNWI: $-15.8 \%$, $p<0.01$ ) compared with B6-control mice. However, there was no significant difference between the two control strains in NSI $(p=0.33)$. In mice that ran during the same 4 weeks of growth, these morphological differences were greater with $\mathrm{A} / \mathrm{J}$-exercise mice showing a significantly narrower Fem.Bicon.Wi $(-7.1 \%$, $p<0.01$ ) and narrower femoral notch width indices (ANWI: $-34.6 \%, p<0.01$; CNWI: $-33.1 \%, p<0.01$; PNWI: $-26.1 \%, p<0.01)$ compared with B6-exercise 


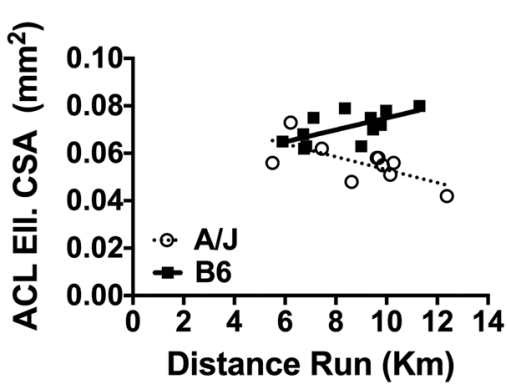

Figure 4. Linear regression showing the relationship between ACL Ell.CSA and daily distance run for both inbred strains after adjusting for body weight. [Color figure can be viewed at wileyonlinelibrary.com]

mice. Additionally, A/J-exercise mice showed a $23.4 \%$ more stenotic femoral notch (NSI: $p<0.01)$ compared with their B6 counterparts. This differential effect between strains is illustrated by the significant strain by treatment interaction for Fem.Bicon.Wi $(F=5.76$, $p=0.02)$, CNWI $(F=2.82, p=0.10)$ and PNWI $(F=6.36$, $p=0.01)$, but not ANWI $(F=0.04, p=0.84)$. The difference in distal femoral morphology between strains that ran is attributed to $\mathrm{A} / \mathrm{J}$-exercise mice having a narrower Fem.Bicon.Wi $(-3.6, p=0.03)$, femoral notch (ANWI: $-9.4 \%, p=0.46$; CNWI: $-9.5 \%, p=0.04$; PNWI: $-6.4 \%, p=0.04)$, and NSI $(-19.1 \%, p<0.01)$ compared with their controls. There was no significant difference in any of the distal femoral morphology measures between B6-exercise mice and their controls. There also was no significant association between the amount A/J and B6 mice ran over the course of 4 weeks and their distal femoral morphology at 10 weeks of age.

Similar to the distal femoral morphology results, $\mathrm{A} / \mathrm{J}$-control mice at 10 weeks of age had a $5.0 \%$ narrower Tib.Bicon.Wi $(p<0.01)$ compared with B6-control mice. AJ-control mice also had less steep posterior tibial plateau slopes (PMTS: $-3.2 \%, p=0.47$; PLTS: $-33.5 \%, p<0.01$ ) compared with their B6 counterparts. In mice that ran, $\mathrm{A} / \mathrm{J}$-exercise mice showed a slight difference in Tib.Bicon.Wi $(1.2 \%, p=0.19)$, and even less steep posterior tibial slopes (PMTS: $-19.2 \%, p<0.01$; PLTS: $-40.3 \%, p<0.01$ ) compared with B6-exercise mice. This differential effect between strains is illustrated by the significant strain by treatment interaction for Tib.Bicon.Wi $(F=22.51$, $p<0.01)$ and PMTS $(F=24.13, p<0.01)$, but not PLTS $(F=0.47, p=0.496)$. The difference in proximal tibial morphology is attributed to $\mathrm{A} / \mathrm{J}$-exercise mice having a $4.6 \%$ less steep PMTS $(p=0.15)$ and $8.2 \%$ less steep PLTS $(p=0.11)$ than their controls, while B6-exercise mice showed a $3.9 \%$ narrower Tib.Bicon.Wi $(p<0.01)$ a $10.2 \%$ steeper PMTS $(p<0.01)$ with a similar PLTS $(p=0.59)$ compared with their controls. There was no significant association between the amount A/J and B6 mice ran and their resulting proximal tibial morphology.

\section{Exercise Effect on Femoral and Tibial ACL Entheseal Morphology}

Compared with B6-control mice at 10 weeks of age, $\mathrm{A} / \mathrm{J}$-control mice had a wider ACL insertion site, as inferred from the femoral and tibial tidemark (TM) measures of the ACL entheses $(8.7 \%, p=0.40$ and $36.9 \%, p<0.01$; respectively). On the femoral side, A/J and B6-control mice had a similar CF.Ar $(p=0.81)$. However, the $\mathrm{CF}$ was heterogeneously distributed at an average $22.3 \%$ shallower depth in $\mathrm{A} / \mathrm{J}$-controls compared with B6-controls (Zones 2-4, $p=0.05-0.002$ ). At the tibial ACL enthesis, the wider TM among A/J-controls resulted in a $33.1 \%$ greater CF.Ar $(p<0.01)$ that, similar to the femoral side, was distributed at an average $22.8 \%$ shallower depth compared with B6-control mice (Zones 3-5, $p=0.07-0.0001$ ). Within strains, exercise had no significant effect on the femoral Fem.TM, CF.Ar, and CF.De present within the enthesis compared with their respective controls (Fig. 5). However, there was a significant strain by treatment interaction for Fem.CF.Ar $(F=5.69, p=0.02)$. Moreover, B6 showed a significant association between the amount they ran and their femoral ACL entheseal morphology, but only in the expanse of their Fem.TM $\left(R^{2}=0.59, p=0.02\right)$.

The results differed within the tibial enthesis. $\mathrm{A} / \mathrm{J}$-exercise mice showed an $18.2 \%$ narrower Tib.TM $(p<0.01)$ with $23.0 \%$ less CF.Ar $(p<0.01)$ and $24.4 \%$ greater average CF.De $(p=0.08)$ compared with their respective controls (Fig. 5). In contrast, the CF.Ar and CF.De of the tibial ACL enthesis of B6-exercise mice were not significantly different than their controls. However, they did exhibit a deeper penetration of the calcified fibrocartilaginous matrix in the central portion of the enthesis (Zones 2-3, $p=0.02-0.009$ ). The strain by treatment interaction was significant for Tib.TM $(F=13.95, p<0.01)$ and CF.Ar $(F=4.99$, $p=0.03)$, but not for average CF.De $(F=0.22$, $p=0.64)$. There was no significant association between the amount $\mathrm{A} / \mathrm{J}$ and $\mathrm{B} 6$ mice ran and their tibial ACL entheseal morphology.

\section{DISCUSSION}

Our data support the first hypothesis that more physiological activity during pubertal growth showed greater morphological differences among traits comprising the ACL-complex in exercised inbred mice compared with their controls, particularly in ACL size and intercondylar femoral notch width. The results also support the second hypothesis that $\mathrm{A} / \mathrm{J}$ and $\mathrm{B} 6$ mice would show a differing ACL-complex phenotype following increased physiological loading during growth. A/ J-exercise mice showed a significantly narrower femoral bicondylar width and intercondylar notch size and shape compared with controls. They also had less steep posterior medial and lateral tibial plateaus, with greater anchorage of the ACL within the anteromedial portion of the tibial enthesis via deeper penetration of the calcified fibrocartilaginous matrix into the bone. In contrast, B6exercise mice demonstrated little difference in knee morphology compared with their controls, with only a narrower tibial bicondylar width and a steeper posterior medial tibial plateau. However, B6-exercise mice did 
A/Js
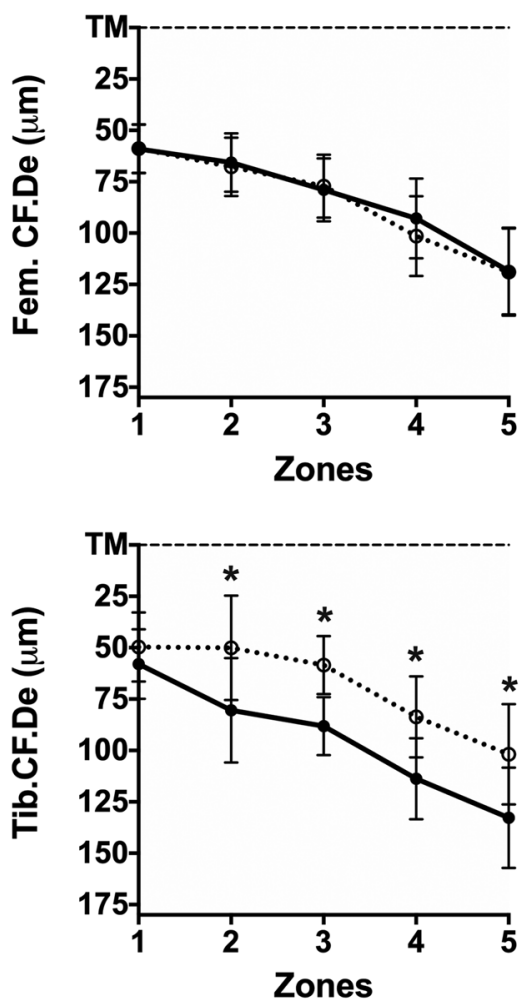

B6s
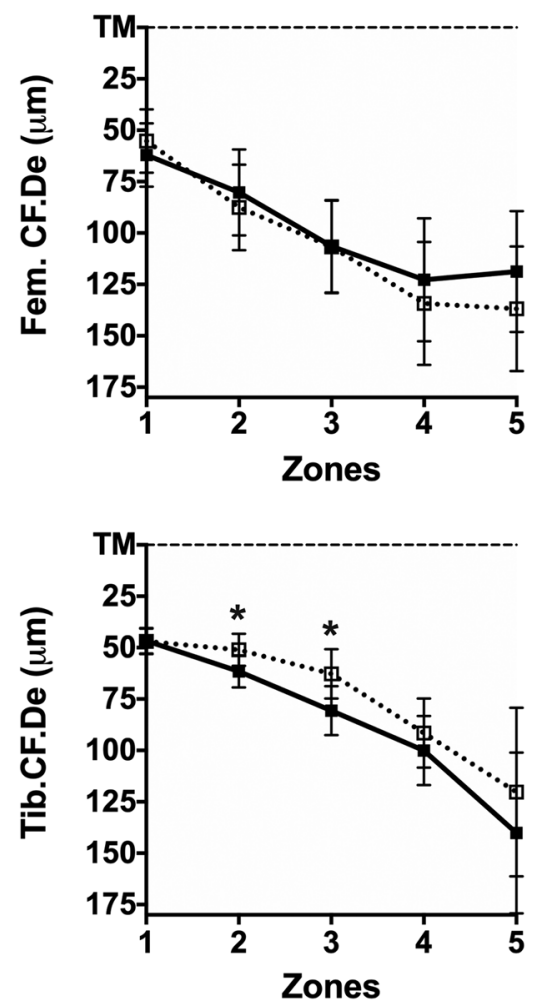

Figure 5. Calcified fibrocartilage depth across the tidemark (TM) for runners and controls of both inbred strains. *Significance at $p<0.05$. [Color figure can be viewed at wileyonlinelibrary.com] show a significantly more hypertrophic ACL compared with controls that were bolstered by deeper penetration of the CF into bone within the central portion of the tibial enthesis.

These outcomes did not support our last hypothesis that the ACL-complex of A/J-exercise mice would be more robust overall than the gracile knee of A/J-control mice. Instead, A/J-exercise mice demonstrated a narrower femoral bicondylar width resulting in a more stenotic intercondylar notch compared with controls. This narrower notch space among A/J-exercised mice may explain why they failed to demonstrate a significantly more hypertrophic ACL than their controls, since notch size may covary with ACL size to mitigate the likelihood of ACL impingement. Not only did A/Jexercise mice show a narrower distal femoral morphology than their controls but they also showed significantly less steep posterior medial and lateral tibial plateaus. Therefore, although A/J-exercised mice did not show a larger ACL Ell.CSA, a less steep posterior tibial slope in A/J-control mice may allow the knee joint of mice that ran to withstand greater physiological loading about the knee and perhaps protect the ACL from increased peak strains by reducing anterior tibial translation ${ }^{49,50}$ compared with the less active controls. Moreover, the broader femoral ACL insertion and significantly deeper penetration of CF within the anteromedial portion of the tibial enthesis in A/J mice that ran may allow the ACL to dissipate applied strains more effectively, while better resisting anterior tibial load during knee extension, ${ }^{51,52}$ compared with their less active controls.

The difference in the ACL-complex between B6control and exercise mice was also unexpected since previously we reported that B6 female mice that ran for 4 weeks during growth showed significantly narrower femoral and humeral diaphyses compared with controls. ${ }^{33}$ Instead, B6-exercise mice showed no difference in the femoral side of the knee joint compared with their controls. The only bony differences observed in B6-exercise mice was a $3.9 \%$ narrower tibial bicondylar width and a $10.2 \%$ steeper medial posterior tibial plateau. Clinically speaking, a very steep medial posterior tibial slope may be viewed as detrimental, however in the case of B6-exercise mice, the steeper medial plateau is more equivalent to that of the lateral plateau slope on (PMTS: $28.7^{\circ} \pm 0.1 .3^{\circ}$, PLTS: $31.4^{\circ} \pm 2.0^{\circ}$, potentially allowing for greater joint stability by reducing the tendency for internal tibial rotation and thus greater ACL peak strains compared with less active controls. A minimization of ACL strain may have also be greater among $\mathrm{B} 6$ mice that ran since they show a $13.5 \%$ larger ACL and deeper penetration of CF within the central portion of the tibial enthesis compared with their less active controls.

It is widely reported that certain anatomical features of the knee joint are associated with increased ACL-injury risk in amateur and professional athletes. ${ }^{49,53}$ Of particular concern is the fact that adolescent females are 1.6 times more likely to suffer 
an ACL injury per athletic exposure compared with adolescent males. ${ }^{54}$ The morphological traits attributed to this disparity are a narrow intercondylar femoral notch, a narrow ACL, and a steep posterior tibial plateau. $^{7,49}$ These traits are widely considered to be non-modifiable. ${ }^{13,15,55}$ Findings from this murine study raise the possibility that these morphological risk factors may in fact be modifiable to some degree during growth, as both A/J and B6 mice exposed to greater physiological loading during puberty showed significant differences within their ACL-complex compared with their genetically similar and age-matched less active controls. However, the outcomes of this research also raise the specter that if morphological traits associated with increased ACL-injury risk are significantly influenced by exercise in children, training regimes would most likely need to be tailored to the individual, since the impact of greater physiological loading is likely dependent on an individual's unique set of traits, as evidenced by the differential differences shown by A/J (gracile knee) and B6 (robust knee) mice that ran throughout puberty.

This is the first study to report that, with exercise during growth, genetically similar inbred mice can show morphological differences in the knee joint that are not present in normal cage activity controls. A strength of this study is the use of voluntary cage-wheel running as the activity perturbation. This mode of activity is physiologically relevant and allows for the detection of subtle effects in a manner that best mimics daily load experience by active teens during growth. Many studies incorporate forced treadmill running to mechanically perturb the musculoskeletal system and is done during working hours when the researcher is present. Voluntary cage-wheel running is cognizant of mouse nocturnal activity patterns and thus their circadian rhythms while minimizing physiological stressors (e.g., shock grid, high-pressure air) that have been linked to elevated corticosterone levels, increased pro-inflammatory cytokines and anxiety. ${ }^{56}$ Most importantly, voluntary cage-wheel running is more consistent with the endurance exercise capacity of mice, which is on average short running bursts of 150 revolutions at speeds that conform to those achieved during treadmill running. ${ }^{57}$ Allowing nocturnal mice to voluntarily exercise resulted in both inbred strains running approximately $9 \mathrm{~km}$ a night throughout the duration of the study. This is far greater than the average one mile per hour rate for $30 \mathrm{~min} /$ day other studies treadmill-exercise animals for to generate a phenotypic response. ${ }^{1,2,58}$ Moreover, our mice ran approximately $250 \mathrm{~km}$ by the completion of the study, compared with less than $100 \mathrm{~km}$ in other studies, and were presumably less stressed while doing so, minimizing physiological and behavioral confounding factors.

However, there are several limitations within this study that caution against the over-interpretation of the results. First, this study did not focus on the cellular bone remodeling activity occurring within the knee joint of mice between the ages of 6 and 10 weeks. Others have shown ${ }^{59}$ that this physiological activity may vary between inbred mouse strains of the same age. For example, it may be that B6 mice demonstrated little bony differences with exercise because their skeletal growth was closer to plateauing than that of $\mathrm{A} / \mathrm{J}$ mice. However, the purpose of our study was to investigate to what extent, if any, exercise had on the ACL-complex in growing mice with phenotypic differences that model the variation among traits comprising the knee joint that are present across humans. Second, the analysis of traits comprising the ACL-complex was performed at the end of the study. Thus, there were no baseline measures of these traits prior to the initiation of the study. This did not allow us to compare developmental changes of specific traits with distance run by each mouse. Since A/J-exercise mice showed a narrower ACL with running, and B6-exercise mice showed a wider ACL with running, it would be important to longitudinally track how these traits develop and are influenced by the underling genetics over time. The differential phenotype of the ACL may be attributed to previously reported metabolic differences that exist between the two mouse strains. ${ }^{60}$ However, for almost every skeletally related trait there was no significant difference between the traits of mice comprising the exercise group in either strain and the number of revolutions they performed each day. This would suggest that the variation in the distance run among mice was within the same activity threshold range for both strains. ${ }^{61}$ Third, we did not mechanically test the tensile strength and stiffness of the ACL, and therefore are unable to determine whether the ACL showed a functional benefit to exercise as Viidik ${ }^{1}$ and Cabaud et al. ${ }^{2}$ observed. Moreover, we do not know whether the greater ACL Ell.CSA shown by B6exercise mice translates to a greater mechanical benefit. Last, the resulting differential phenotypes shown in the exercise cohorts were only shown in female mice. It is unknown if males, which voluntarily run less on a daily basis $^{57}$ would show similar differences following exercise.

In conclusion, this study contributes to the discussion as to whether certain biological risk factors known to correlate with ACL injury are modifiable during growth. Our data confirmed our hypothesis that mice that run voluntarily during pubertal growth show a different knee morphology compared with those with normal cage activity. Moreover, we confirmed our hypothesis that the differences between $\mathrm{A} / \mathrm{J}$ and B6 female control mice knee morphology would continue to differ in significantly new ways following 4 weeks of exercise during growth. Neither outcome has been previously reported. Collectively our findings suggest it may be possible to modify certain morphological traits comprising the ACL-complex during growth; however, the intervention chosen to adaptively direct a desired change is likely to be individual specific. 


\section{AUTHORS' CONTRIBUTIONS}

Study design: S.H.S., B.T.N., E.M.W., and J.A.A.M. Data collection: S.H.S., C.T.M., D.N.O., and B.T.N. Data interpretation and manuscript preparation: S.H.S., C.T.M., D.N.O., E.M.W., and J.A.A.M. Manuscript approval: S.H.S., C.T.M., D.N.O., B.T.N., E.M.W., J.A.A.M.

\section{ACKNOWLEDGMENT}

We would like to thank Dr. Melanie L. Beaulieu and Rebecca Falzon for comments and suggestions on earlier drafts of this manuscript.

\section{ORCID}

\section{StephenH Schlecht (D) http://orcid.org/0000-0002-} 6857-927X

\section{REFERENCES}

1. Viidik A. 1968. Elasticity and tensile strength of the anterior cruciate ligament in rabbits as influenced by training. Acta Physiol Scand 74:372-380.

2. Cabaud HE, Chatty A, Gildengorin V, et al. 1980. Exercise effects on the strength of the rat anterior cruciate ligament. Am J Sports Med 8:79-86.

3. Grzelak P, Podgorski M, Stefanczyk L, et al. 2012. Hypertrophied cruciate ligament in high performance weightlifters observed in magnetic resonance imaging. Int Orthop 36:1715-1719.

4. Chandrashekar N, Slauterbeck J, Hashemi J. 2005. Sexbased differences in the anthropometric characteristics of the anterior cruciate ligament and its relation to intercondylar notch geometry. Am J Sports Med 33:1492-1498.

5. Brandon M, Haynes P, Bonamo J, et al. 2006. The association between posterior-inferior tibial slope and anterior cruciate ligament insufficiency. Arthroscopy 22:894-899.

6. Giffin J, Vogrin T, Zantop T, et al. 2004. Effects of increasing tibial slope on the biomechanics of the knee. Am J Sports Med 32:376-382.

7. Lipps D, Oh Y, Ashton-Miller J, et al. 2012. Morphologic characteristics help explain the gender difference in peak anterior cruciate ligament strain during a simulated pivot landing. Am J Sports Med 40:32-40.

8. Lipps D, Wojtys E, Ashton-Miller J. 2013. Anterior cruciate ligament fatigue failures in knees subjected to repeated simulated pivot landings. Am J Sports Med 41:1058-1066.

9. Chaudhari A, Zelman E, Flanigan D, et al. 2009. Anterior cruciate ligament-injured subjects have smaller anterior cruciate ligaments than matched controls: a magnetic resonance imaging study. Am J Sports Med 37:1282-1287.

10. Keays S, Keays R, Newcombe P. 2016. Femoral intercondylar notch width size: a comparison between siblings with and without anterior cruciate ligament injuries. Knee Surg Sports Traumatol Arthrosc 24:672-679.

11. Simon R, Everhart J, Nagaraja H, et al. 2010. A case-control study of anterior cruciate ligament volume, tibial plateau slopes and intercondylar notch dimensions in ACL-injured knees. J Biomech 43:1702-1707.

12. Beynnon B, Hall J, Sturnick D, et al. 2014. Increased slope of the lateral tibial plateau subchondral bone is associated with greater risk of noncontact ACL injury in females but not in males. Am J Sports Med 42:1039-1048.

13. Bojicic KM, Beaulieu ML, Imaizumi krieger DY, et al. 2017. Association between lateral posterior tibial slope, body mass index, and ACL injury risk. Orthop J Sports Med 5:7.

14. Tuca M, Hayter C, Potter H, et al. 2016. Anterior cruciate ligament and intercondylar notch growth plateaus prior to cessation of longitudinal growth: an MRI observational study. Knee Surg Sports Traumatol Arthrosc 24:780-787.

15. Price M, Tuca M, Cordasco F, et al. 2017. Nonmodifiable risk factors for anterior cruciate ligament injury. Curr Opin Pediatr 29:55-64.

16. Bass SL, Saxon L, Daly RM, et al. 2002. The effect of mechanical loading on the size and shape of bone in pre-, peri-, and postpubertal girls: a study in tennis players. J Bone Miner Res 17:2274-2280.

17. Macdonald H, Kontulainen S, MacKelvie-O'Brien K, et al. 2005. Maturity- and sex-related changes in tibial bone geometry, strength and bone-muscle strength indices during growth: a 20-month pQCT study. Bone 36:1003-1011.

18. Moro M, van der Meulen M, Kiralti BJ, et al. 1996. Body mass is the primary determinant of midfemoral bone acquisition during adolescent growth. Bone 19:519-526.

19. Kanehisa H, Ikegawa S, Tsunoda N, et al. 1994. Crosssectional areas of fat and muscle in limbs during growth and middle age. Int J Sports Med 15:420-425.

20. Rogol A, Roemmich J, Clark P. 2002. Growth at puberty. J Adolesc Health 31:192-200.

21. Ruff C. 2003. Growth in bone strength, body size, and muscle size in a juvenile longitudinal sample. Bone 33:317-329.

22. Dowling B, Dart A. 2005. Mechanical and functional properties of the equine superficial digital flexor tendon. Vet J 170:184-192.

23. O’Brien FJ, Hardiman DA, Hazenberg JG, et al. 2005. The behaviour of microcracks in compact bone. Eur J Morphol 42:71-79.

24. Daly RM, Saxon L, Turner CH, et al. 2004. The relationship between muscle size and bone geometry during growth and in response to exercise. Bone 34:281-287.

25. Kasashima Y, Smith R, Birch H, et al. 2002. Exercise-induced tendon hypertrophy: cross-sectional area changes during growth are influenced by exercise. Equine Exerc Physiol 34:264-268.

26. Kontulainen S, Sievanen H, Kannus P, et al. 2003. Effect of long-term impact-loading on mass, size, and estimated strength of humerus and radius of female racquet-sports players: a peripheral quantitative computed tomography study between young and lod starters and controls. J Bone Miner Res 18:352-359.

27. Vicente-Rodriguez G, Jimenez-Ramirez J, Ara I, et al. 2003. Enhanced bone mass and physical fitness in prepubescent footballers. Bone 33:853-859.

28. Vicente-Rodriguez G. 2005. How does exercise affect bone development and growth. Sports Med 36:561-569.

29. Carballo C, Hutchinson I, Album Z, et al. 2018. Biomechanics and microstructural analysis of the mouse knee and ligaments. J Knee Surg 31:520-527.

30. Wang V, Banack T, Tsai C, et al. 2006. Variability in tendon and knee joint mechanics among inbred mouse strains. J Orthop Res 24:1200-1207.

31. Beaulieu M, Carey G, Schlecht S, et al. 2015. Quantitative comparison of the microscopic anatomy of the human ACL femoral and tibial entheses. J Orthop Res 33:1811-1817.

32. Beaulieu M, Carey G, Schlecht SH, et al. 2016. On the heterogeneity of the femoral enthesis of the human ACL: microscopic anatomy and clinical implications. J Exper Orthop 3:14.

33. Schlecht S, Ramcharan MA, Yang Y, et al. 2018. Differential adaptive response of growing bones from two female inbred mouse strains to voluntary cage-wheel running. J Bone Miner Res Plus 2:143-153.

34. Black B, Croom J, Eisen E, et al. 1998. Differential effects of fat and sucrose on body composition in $\mathrm{A} / \mathrm{J}$ and $\mathrm{C} 57 \mathrm{BL} / 6$ mice. Metabol 47:1354-1359.

35. Gallou-Kabani C, Vige A, Gross M-S, et al. 2007. C57BL/6J and A/J mice fed a high-fat diet delineate components of metabolic syndrome. Obesity 15:1996-2005. 
36. Price C, Herman BC, Lufkin T, et al. 2005. Genetic variation in bone growth patterns defines adult mouse bone fragility. $\mathrm{J}$ Bone Miner Res 20:1983-1991.

37. Schlecht SH, Smith LM, Ramcharan MA, et al. 2017. Canalization leads to similar whole bone mechanical function at maturity in two inbred strains of mice. J Bone Miner Res 32:1002-1013.

38. Hagenauer M, Perryman J, Lee T, et al. 2009. Adolescent changes in the homeostatic and circadian regulation of sleep. Dev Neurosci 31:276-284.

39. Kercmar J, Tobet S, Majdic G. 2014. Social isolation during puberty affects female sexual behavior in mice. Front Behav Neurosc 8:337.

40. Dutta S, Sengupta P. 2016. Men and mice: relating their ages. Life Sci 152:244-248.

41. Fitch R, Montgomery R, Milton J, et al. 1995. The intercondylar fossa of the normal canine stifle an anatomic and radiographic study. Vet Surg 24:148-155.

42. Comerford E, Tarlton J, Avery N, et al. 2006. Distal femoral intercondylar notch dimensions and their relationship to composition and metabolism of the canine anterior cruciate ligament. Osteoarthr Cartilage 14:273-278.

43. Reif U, Probst C. 2003. Comparison of tibial plateau angles in normal and cranial cruciate deficient stifles of Labrador Retrievers. Vet Surg 32:385-389.

44. Evans EJ, Benjamin M, Pemberton DJ. 1990. Fibrocartilage in the attachment zones of the quadriceps tendon and patellar ligament of man. J Anat 171:155-162.

45. Morrison J. 1969. Function of the knee joint in various acivities. Biomed Eng 4:573-580.

46. Morrison J. 1970. The mechanics of the knee joint in relation to normal walking. J Biomech 3:51-61.

47. Chaudhari A, Andriacchi T. 2006. The mechanical consequences of dynamic frontal plane limb alignment for noncontact ACL injury. J Biomech 39:330-338.

48. Hewett TE, Myer G. 2011. The mechanistic connection between the trunk, knee, and anterior cruciate ligament injury. Exerc Sport Sci Rev 39:161-166.

49. Wojtys E, Beaulieu M, Ashton-Miller J. 2016. New perspectives on ACL injury: on the role of repetitive submaximal knee loading causing ACL fatigue failure. J Orthop Res 34:2059-2068.
50. Yamaguchi K, Cheung E, Mathew J, et al. 2017. ACL force and knee kinematics after posterior tibial slope-reducing osteotomy. Orthop J Sports Med 5(suppl 6):1-2. https://doi. org/10.1177/2325967117S00308

51. Benjamin M, Toumi H, Ralphs JR, et al. 2006. Where tendons and ligaments meet bone: attachment sites ('entheses') in relation to exercise and/or mechanical load. J Anat 208:471-490.

52. Girgis F, Marshall J, Monajem A. 1975. The cruciate ligaments of the knee joint. Anatomical, functional and experimental analysis. Clin Orthop Relat Res 106:216-231.

53. Sturnick D, Vacek P, DeSarno M, et al. 2015. Combined anatomic factors predicting risk of anterior cruciate ligament injury for males and females. Am J Sports Med 43:839-847.

54. Gornitzky A, Lott A, Yellin J, et al. 2017. Sport-specific yearly risk and incidence of anterior cruciate ligament tears in high school athletes: a systematic review and meta-analysis. Pediatrics 140:109-109.

55. Weber A, Bach B Jr., Bedi A. 2017. How do we eliminate risk factors for ACL injury? In: Musahl V, Karlsson J, Kuroda R., Zaffagnini S., editors. Rotatory knee instability. Switzerland: Springer; p 465-472.

56. Svensson M, Rosvall P, Boza-Serrano A, et al. 2016. Forced treadmill exercise can induce stress and increase neuronal damage in a mouse model of global cerebral ischemia. Neurobiol Stress 5:8-18.

57. De Bono J, Adlam D, Paterson D, et al. 2006. Novel quantitative phenotypes of exercise training in mouse models. Am J Physiol Regulat Integr Comp Physiol 290:R926-R934.

58. Tipton C, Schild R, Tomanek R. 1967. Influence of physical activity on the strength of knee ligaments in rats. Am J Physiol 212:783-787.

59. Poliachik SL, Threet D, Srinivasan S, et al. 2008. 32 wk old $\mathrm{C} 3 \mathrm{H} / \mathrm{HeJ}$ mice actively respond to mechanical loading. Bone 42:653-659.

60. Hall D, Poussin C, Velagapudi V, et al. 2010. Peroxisomal and microsomal lipid pathways associated with resistance to hepatic steatosis and reduced pro-inflammatory state. J Biol Chem 285:31011-31023.

61. Rubin CT, Lanyon LE. 1984. Dynamic strain similarity in vertebrates: an alternative to allometric limb bone scaling. J Theoret Biol 107:321-327. 